ORIGINAL ARTICLE

\title{
A survey of current consultant practice of treatment of severe ankle sprains in emergency departments in the United Kingdom
}

\author{
M W Cooke, S E Lamb, J Marsh, J Dale
}

Emerg Med J 2003;20:505-507

See end of article for authors' affiliations

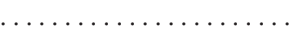

Correspondence to: Dr M Cooke, Centre for Primary Health Care Studies, University of Warwick, Gibbett Hill Road, Coventry CV4 7AL UK; matthew.cooke@ warwick.ac.uk

Accepted for publication 3 December 2002
Objective: To determine current consultant practice in larger UK emergency departments in the management of severe ankle sprains.

Design: Questionnaire study to all UK emergency departments seeing more than 50000 new patients per year.

Results: $70 \%$ response rate. Most popular treatment was ice, elevation, Tubigrip, and exercise, each of which was reported as used in most cases by over $70 \%$ of respondents. Crutches, early weight bearing, and non-steroidal anti-inflammatory drugs were each reported as used in most cases at over half of responding departments. Physiotherapy was usually only used in selected cases. Rest was usually advised for one to three days (35\%). Follow up was only recommended for selected patients.

Conclusions: The results of this survey suggest that there is considerable variation in some aspects of the clinical approach (including drug treatment, walking aids, periods of rest) taken to the management of severe ankle sprains in the UK, although in some areas (for example, not routinely immobilising, early weight bearing as pain permits, use of physiotherapy, use of rest, ice, and elevation) there was concordance.
S prains of the lateral ligaments of the ankle joint account for between $3 \%$ and $5 \%$ of all emergency department (ED) attendances in the UK, ${ }^{1}$ with about 5600 injuries each day. ${ }^{2}$ An incidence of 1 per 10000 people per day has been suggested. ${ }^{3}$ It is probable that many more attend primary care facilities, such as minor injury services, general practitioners, and sports clinics. The injury is painful and incapacitating, and, for all but the most minor weight bearing is difficult to tolerate. Activities of daily living can be significantly compromised in the first few weeks, and although acute symptoms resolve, persistent symptoms are reported to occur in $30^{4}$ to $40 \%{ }^{5}$ of people. These include instability or giving way of the ankle, swelling, unsightly appearance, and pain. Recurrent sprains often occur.

The optimal management for severe sprains is unclear and recommendations range from no intervention, to physiotherapy, different types of brace, or surgical repair of the ligaments. A Cochrane review of ankle sprain treatment concluded that functional treatment seems to be the favourable strategy for treating acute ankle sprains when compared with immobilisation. ${ }^{6}$ However, the results should be interpreted with caution, as most of the differences are not significant after exclusion of the low quality trials. Many trials were poorly reported and there was variety among the functional treatments evaluated. A further Cochrane review concluded that there is insufficient evidence to determine the relative effectiveness of surgical and conservative treatment for acute injuries of the lateral ligament complex of the ankle. $^{7}$

The aim of this study was to undertake a survey of national practice in EDs to inform the design of a randomised controlled trial of treatment of acute ankle sprains.

\section{METHODS}

All 183 EDs in United Kingdom seeing more than 50000 new patients were identified from the British Association for Accident and Emergency Medicine Directory 2001/2002 ${ }^{6}$ and the lead consultants were sent a brief questionnaire (appendix) to ascertain the current methods of treatment used in a typical severe ankle injury. Replies were anonymous and therefore follow up and analysis of non-responders was not possible. A copy of the appendix is available on the journal web site (http://www.emjonline.com/supplemental).

Severe ligamentous injury (grade III) indicates incompetence of the anterior talofibular ligament, demonstrated by the anterior drawer sign. However, clinical grading of injury is often impossible in the emergency situation because the pain and swelling prevents full clinical assessment, therefore the questionnaire presented a clinical scenario together with a series of questions to ascertain key characteristics of the clinical protocols used at each hospital. The scenario was intended to describe a patient with a typical severe ankle injury: "A 40 year old rugby player has just injured his ankle today. It is bruised and very swollen over the lateral ligament. There is no apparent instability but any ankle movement is very painful. He cannot weight bear. The x-ray shows no abnormality". Questions inquired about the usual form of treatment prescribed at the ED for the injury; other treatments used in selected cases; advice on rest, weight bearing and exercises; timing of application of bracing if used; use of physiotherapy and magnetic resonance imaging; use of follow up clinics, orthopaedic and sports injury services. Descriptive data were generated to identify the most frequently used treatments, combination of treatments, type and timing of advice, and follow up options.

\section{RESULTS}

The response was $70.3 \%$ ( 83 of 118 ).

\section{Routine treatment of severe ankle sprain}

Results are given in table 1 , as percentages of replies in a category for each treatment. Most popular choices of treatment were ice, elevation, Tubigrip, and exercise, all of which were chosen as treatment for "most cases" by over 


\begin{tabular}{|llllll|}
\hline $\begin{array}{l}\text { Table 1 Choice of treatments for ankle injury reported by A\&E consultants (\% of } 83 \\
\text { respondents) }\end{array}$ & \multicolumn{5}{l|}{} \\
\hline \multicolumn{5}{c}{ Most cases } \\
\hline $90 \%$ & Selected cases & Rarely $<10 \%$ & Never & No answer \\
\hline Elevation & 90.4 & 3.6 & 1.2 & 1.2 & 3.6 \\
lce & 80.7 & 6.0 & 6.0 & 6.0 & 1.2 \\
Tubigrip & 73.5 & 14.5 & 3.6 & 6.0 & 2.4 \\
Crepe bandage & 2.4 & 10.8 & 27.7 & 51.8 & 7.2 \\
Strapping & 1.2 & 6.0 & 18.1 & 67.5 & 7.2 \\
POP cast & 3.6 & 25.3 & 44.6 & 21.7 & 4.8 \\
Aircast splint & 0.0 & 7.2 & 13.3 & 72.3 & 7.2 \\
Flexible cast & 0.0 & 4.8 & 14.5 & 73.5 & 7.2 \\
Bledsoe boot & 0.0 & 0.0 & 15.7 & 73.5 & 10.8 \\
Walking stick & 2.4 & 33.7 & 30.1 & 15.7 & 18.1 \\
Crutches & 55.4 & 37.3 & 3.6 & 1.2 & 2.4 \\
Exercises & 75.9 & 15.7 & 1.2 & 0.0 & 7.2 \\
Rest & 8.4 & 15.7 & 31.3 & 31.3 & 13.3 \\
Weight bearing & 49.4 & 25.3 & 10.8 & 3.6 & 10.8 \\
Physiotherapy & 22.9 & 69.9 & 2.4 & 2.4 & 2.4 \\
NSAIDs & 56.6 & 30.1 & 7.2 & 2.4 & 3.6 \\
NSAID gel & 2.4 & 8.4 & 28.9 & 50.6 & 9.6 \\
MRI & 0.0 & 0.0 & 8.4 & 90.4 & 1.2 \\
\hline
\end{tabular}

Table 2 Advice for rest and exercise that A\&E consultants reported they would offer a patient presenting as in the scenario (\% of 83 respondents)

\begin{tabular}{lllllll}
\hline Advice & $<\mathbf{1}$ day & $\mathbf{1 - 3}$ days & $\mathbf{3 - 7}$ days & $\mathbf{1 - 3}$ weeks & As pain permits & No answer \\
\hline Rest & 7.2 & 34.9 & 9.6 & 8.4 & 12.0 & 27.7 \\
Weight bearing & 2.4 & 12.0 & 3.6 & 2.4 & 71.1 & 8.4 \\
$\begin{array}{l}\text { Non-weight } \\
\text { bearing exercises }\end{array}$ & 55.4 & 3.6 & 1.2 & 1.2 & 27.7 & 10.8 \\
\hline
\end{tabular}

$70 \%$ of respondents. Other common choices were crutches, early weight bearing and non-steroidal anti-inflammatory drugs, chosen as treatment for "most cases" by over $50 \%$ of respondents. The routine use of flexible casts, plaster of Paris casts, and other forms of strapping was less than 5\%. Many departments opted to use a method of rigid support in selected cases, and in this situation plaster of Paris casting was the most popular choice, being used by $25 \%$ of departments. Bledsoe boots were used infrequently (15.7\% of departments reported using it rarely). Physiotherapy was usually only used for selected cases.

\section{Routine advice for severe ankle sprain}

Responses given on the questionnaire to the questions that asked about the advice that would be offered to a patient presenting with an ankle injury of the type described in the scenario are given in table 2 . In advising a rest period after an ankle injury, most consultants were fairly conservative, with the majority (35\%) opting for a rest period of one to three days. The majority of consultants opt to prescribe non-weight bearing exercises within a 24 hour period of the injury, and to begin weight bearing as pain permitted $(71 \%)$. Forty per cent of consultants reported applying a mechanical brace (POP cast, aircast splint, or flexible cast) in most or selected cases. Twenty eight per cent of these advocated a delay period before applying the brace. The remainder applied the brace immediately. Too few advice $(n=4)$ sheets were returned to undertake any analysis.

\section{Follow up options}

Most consultants reported that follow up was only advised for selected cases (table 3), and when this was offered it was mostly to an emergency department or a physiotherapy clinic (44\% and 47\% respondents respectively). Emergency department clinic follow up for most cases was reported by $27 \%$ respondents. Referral to a general fracture or orthopaedic, sports or specialist ankle clinic was rare, $69 \%$ never using or not having access to a sports clinic, and $81 \%$ never using or not having access to a specialised ankle clinic.

Table 3 Follow up actions for severe ankle sprain reported by A\&E consultants (\%)

\begin{tabular}{llllll}
\hline & $\begin{array}{l}\text { Most cases } \\
\mathbf{9 0 \%}\end{array}$ & $\begin{array}{l}\text { Selected } \\
\text { cases }\end{array}$ & $\begin{array}{l}\text { Rarely } \\
<10 \%\end{array}$ & Never & No reply \\
\hline $\begin{array}{l}\text { A\&E clinic } \\
\text { General fracture/orthopaedic }\end{array}$ & 27.7 & 44.6 & 20.5 & 2.4 & 4.8 \\
clinic & 2.4 & 10.8 & 41.0 & 30.1 & 15.7 \\
Physiotherapist run clinic & 9.6 & 47.0 & 7.2 & 25.3 & 10.8 \\
Sports clinic & 1.2 & 1.2 & 10.8 & 69.9 & 16.9 \\
Specialist ankle clinic & 1.2 & 0.0 & 0.0 & 81.5 & 17.3 \\
\hline
\end{tabular}




\section{DISCUSSION}

The results of this survey suggest that there is considerable variation in some aspects of the clinical approach (including drug treatment, walking aids, periods of rest) taken to the management of severe ankle sprains in the UK, although in some areas (for example, not routinely immobilising, early weight bearing as pain permits, use of physiotherapy, use of rest, ice, and elevation) there was concordance. The findings need to be treated with some caution as the study is based on the opinion of lead clinicians, not on actual practice. They are also the practice of larger departments (over 50000 new patients per year); smaller departments may have less access to facilitis and resources, for example, physiotherapy, magnetic resonance imaging. The extent to which clinicians within each department adhere to the reported departmental practice is unknown. However, the commonest treatment that was advocated is rest until pain subsides, followed by mobilisation, early non-weight bearing exercise, ice, elevation, Tubigrip, oral non-steroidal anti-inflammatory drugs, and follow up only in selected cases.

There is a lack of high quality evidence to support clinical decisions for one type of treatment above another particularly in severe sprains. A recently published meta-analysis of relevant trials ${ }^{2}$ has revealed that treatment with a plaster cast was significantly worse than treatment with Tubigrip or bracing, with respect to subsequent giving way and residual pain at six weeks. However, all types of functional treatment were classed together in the meta-analysis, and it was not possible to draw distinctions between them. One trial of mild to moderate ankle sprains (grade 1 and 2 ) revealed more pain at one week for those treated with Tubigrip but failed to reveal any other differences, and follow up was limited to one week. ${ }^{9}$ There have been no randomised studies to support the use of Bledsoe boot type immobilisation. No reliable studies have been found that describes long term outcome for varying treatments.

Present evidence suggests that functional treatment is better than either immobilisation over three weeks or no treatment. There remains a need for a well conducted and adequately powered randomised controlled trial of the clinical and cost effectiveness of different clinical approaches $^{10}$ before evidence based guidelines can be written. This survey has been used to inform decisions about the design of a randomised controlled trial $(n=1500)$ to compare Tubigrip, dynamic splinting, plaster of Paris cast, and Bledsoe boot.

\section{Contributors}

MWC designed the questionnaire and managed its distribution and results collation SL assisted with data collation, design and analysis of questionnaire, preparing sections of first draft of paper, JM cleaned, analysed and summarised the questionnaire data JD contributed to the design of the study, the interpretation of the findings and the drafting of the paper. All authors were involved in writing the final paper. MWC is the guarantor of the paper.

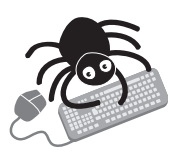

A copy of the questionnaire used in this study is available to view on the journal web site (http://www.emjonline.com/supplemental)

\section{Authors' affiliations}

M W Cooke, J Marsh, J Dale, Centre for Primary Health Care Studies, University of Warwick, UK

S E Lamb, Interdisciplinary Research Centre in Health, Coventry

University, UK

Funding: none.

Conflicts of interest: none declared.

\section{REFERENCES}

1 Trundle HR. Physiotherapy: the contribution. In: Skinner D, Swain A, Peyton R, eds. Cambridge textbook of accident and emergency medicine. Cambridge: Cambridge University Press, 1997:814.

2 Pijnenburg AC, Van Dijk CN, Bossuyt PM, et al. Treatment of ruptures of the lateral ankle ligaments: a meta-analysis. J Bone Joint Surg [Am] 2000;82:761-73.

3 Kannus $P$, Renström $P$. Treatment for acute tears of the lateral ligaments of the ankle. J Bone Joint Surg 1991;73-A:305-12.

4 Schaap GR, de Keizer G, Marti K. Inversion trauma of the ankle. Arch Orthop Trauma Surg 1989;108:273-5.

5 Geber JP, Williams GN, Scoville CR, et al. Persistent disability associated with ankle sprains: a prospective examination of an athletic population. Foot Ankle Int 1998:653-60

6 Kerkhoffs GMMJ, Rowe BH, Assendelft WJJ, et al. Immobilisation and functional treatment for acute lateral ankle ligament injuries in adults (Cochrane review). Cochrane Library. Issue 3. Oxford: Update Software 2000.

7 Kerkhoffs GMMJ, Handoll HHG, de Bie R, et al. Surgical versus conservative treatment for acute injuries of the lateral ligament complex of the ankle in adults (Cochrane review). Cochrane Library., Issue 3. Oxford: Update Software, 2002.

8 British Association of Accident and Emergency Medicine. BAEM directory. London: BAEM, 2000.

9 Watts BL, Armstrong B. A randomised controlled trial to determine the effectiveness of double Tubigrip in grade 1 and 2 (mild to moderate) ankle sprains. Emerg Med J 2001;18:46-50.

10 Wilson S, Cooke MW. Double bandaging of ankles. BMJ 1998;317:1722-3. 